\title{
Sticks and stones: Associative learning alone?
}

\section{Jennifer Vonk ${ }^{1}$}

Published online: 3 July 2019

(C) The Psychonomic Society, Inc. 2019

\section{Summary}

Gruber et al. (Current Biology, 29, 686-692, 2019) report that New Caledonian crows engage in mental representation to solve a problem involving a tool. Although the crows' success is impressive, an associative account of their behavior calls into question the extent to which the data reflect representation of future states.

Keywords Crows $\cdot$ Tool-use $\cdot$ Planning $\cdot$ Associative

Gruber et al. (2019) report that crows are able to correctly anticipate future steps in a problem-solving sequence involving tools, even when each stage of the problem was out of view. They argue that the results indicate that crows can plan.

Despite the fact that planning abilities are telling of important cognitive capacities such as metarepresentation, representation of the future, cognitive regulation, etc., the topic has not been well studied within comparative cognition. Like many other exciting topics of study, the study of planning suffers from the challenge that it is difficult to tease apart an associative account whereby animals have learned that particular responses or objects are associated with future rewards and an account by which they can represent actions that might be useful in the future contingent upon circumstances they have not yet experienced. As Hampton (2018) suggested, that animals predict and choose current behaviors in anticipation of future rewards should not be surprising. It is more interesting to determine if they plan flexibly such that they work to procure specific tools only under circumstances where that future tool would be useful and not when it would not be. Seed and Dickerson (2016) also acknowledged that it is crucial for animals to anticipate future outcomes that differ from events that have occurred in their past in order for researchers to confidently determine that they represent future states. In Gruber and colleagues' study, the crows have experienced all of the outcomes prior to each test, so it is challenging to determine whether they are reasoning about future versus past states.

Jennifer Vonk

vonk@oakland.edu

1 Department of Psychology, Oakland University, Rochester, MI, USA
Most readers will likely be fatigued by yet another diatribe on the importance of distinguishing between "associative" and "cognitive" accounts of apparently complex behaviors, and to some degree, this may be a false dichotomy. But a serious problem persists in comparative psychology, with researchers continuing to over-interpret interesting patterns of behavior. In doing so, they often minimize the significance of the actual behavior because readers are distracted by the human-like trait the study is intending to reveal and neglect to ask (and answer) deeper questions about what the behavior actually reveals about nonhuman cognition. Researchers would be better served by focusing on the exact cognitive processes underlying apparently complex behavior in other species, rather than by trying to find ways to attribute human-like processes to species that have faced very different selection pressures in their evolutionary history.

There is no doubt that the crows' performance is incredibly impressive. They are able to represent multiple aspects of the overall problem, even when they cannot view all of the elements at once. However, each problem may be scaffolded on previous learning such that the crows have learned which object works in which apparatus. They do not have to imagine a scenario in which the tool or stone would not work and modify it, or choose a different object. Only one free object is presented initially and it is clear from previous training which object will work in the final apparatus that contains the food. Only the stick works in the tube and only the stone works in the platform. In the first two experiments, the crows can follow the rule of always releasing the object that differs from the object they currently have, except in the shortcut condition, which is designed to demonstrate that they are representing the final stage of the problem. In this condition, the substage involves the same tool to be released from two 
different apparatuses, but this tool cannot be used in the final stage, and so should be ignored. The birds may have succeeded in this condition because there was a stronger positive association between the stone and the platform containing the food (in the final stage) than with the stone and the platform or tube containing the stick (in the substage). That is, if crows have a stone in their possession and can choose to act on a tube with a stick, a platform with a stick, and a platform with food, the strongest positive associative response would be to the platform with the food because the crows were never directly rewarded for releasing the stick, but they were directly rewarded for using the stone in the platform with food.

It is also clear in Experiment 3, based on their previous training, which apparatus will work with the one tool they have been given. Although crows could no longer use the rule "work to procure the tool not currently in possession," because both tools in the subgoal stage meet this criteria, from an associative account, it is not surprising that crows bypassed nonfunctional apparatuses in order to use the tool in the one apparatus in which that tool had been used successfully in the past. That is, again, sticks work in tubes and stones work in platforms. In every experiment where animals must infer a solution, there must be observable cues that allow them to do so. In this case, the cues are tied to past rewards rather than abstract representations of future states, which is the same challenge that comparative psychologists have struggled with for decades (e.g., Vonk \& Povinelli, 2006). It is unclear what planning is involved in Experiment 3 because the crows can see which apparatus would not work with the tool they have in hand, making the incorrect choice not that distracting.

It is likely that the crows would fail if they had to choose which tool to bring to the second stage of the problem. Despite both tasks requiring mental representation, choosing which apparatus to take a tool from, rather than choosing between two tools in the same apparatus, appears to have been much more cognitively demanding in the current study, possibly due to the increased working memory load this task required. This is reminiscent of previous work with apes and human children. Both chimpanzees and children fail when choosing between tools versus directly acting with one option available to them as in trap table experiments (Girndt, Meier, \& Call, 2008). This difficulty likely involves a failure of inhibition.
The crows perform better when using a stick to dislodge a stone than vice versa - probably because they are accustomed to using sticks to manipulate objects, such as building nests and hunting for insects. However, overall, many crows did not pass, indicating the possibility that the task may not reflect a simple associative learning exercise. Only some of the birds exhibited a pattern where they learned the correct response and then stuck with it consistently. Many of the birds seemed to be responding randomly and at chance. A more careful analysis of the patterns of success and failures may be more informative than a focus on the few succeeding subjects alone.

We can learn much about crow cognition from the paradigm. We can learn what they are capable of learning based on past experience, how they manipulate and use tools, and probably something about inhibition and cognitive regulation. But whether we can learn about future planning is debatable.

\section{References}

Girndt, A., Meier, T., \& Call, J. (2008). Task constraints mask great apes' ability to solve the trap-table task. Journal of Experimental Psychology: Animal Behavior Processes, 34(1), 54-62. https://doi. org/10.1037/0097-7403.34.1.54

Gruber, R., Schiestl, M., Boeckle, M., Gray, R.D., Clayton, N.S., \& Taylor, A.H., (2019). New Caledonian crows use mental representations to solve metatool problems. Current Biology, 29, 686-692.

Hampton, R. (2018). Parallel overinterpretation of behavior of apes and corvids. Learning \& Behavior. https://doi.org/10.3758/s13420-0180330-5

Seed, A.M. \& Dickerson, K.L. (2016). Future thinking: Children but not apes consider multiple possibilities. Current Biology, 26, R523R542.

Vonk, J. \& Povinelli, D.J. (2006). Similarity and difference in the conceptual systems of primates: The unobservability hypothesis. In E.Wasserman and T. Zentall (Eds.). Comparative cognition: Experimental explorations of animal intelligence. [pp. 363-387]. Oxford, UK, Oxford University Press.

Publisher's note Springer Nature remains neutral with regard to jurisdictional claims in published maps and institutional affiliations. 